\title{
Psychometric properties of three personality inventories translated to
}

\author{
Sinhalese
}

Lakal Dissabandara, Natalie Loxton, Shavindra Dias, Mark Daglish, Alfreda Stadlin

\section{Background}

Personality has been increasingly recognized as closely associated with predisposition to develop a wide range of disease conditions. However, at present, in Sri Lanka, there is a lack of awareness of the importance of personality in research and in clinical practice, largely due to the unavailability of tools measuring personality in native languages. Behavioural inhibition system behavioural activation system (BIS/BAS) scales and sensitivity to punishment and sensitivity to reward questionnaire (SPSRQ) which are based on Gray's theory of personality and the sensation seeking scale (SSS), which is based on Zuckerman's sensation seeking theory have been commonly used in both research and clinical practice. Aims

The aim of the study is to translate and assess the psychometric properties of a Sinhalese adaptation of the BIS/BAS, SPSRQ and SSS scales in a Sri Lankan population

Methods

The BIS/BAS, SPSRQ and SSS were translated into Sinhalese according to standard method and administered to a group of two hundred and thirty three Sri Lankan university students. Confirmatory factor analysis (CFA) and reliability analysis were used to evaluate the translated versions.

Results

CFA and reliability analysis revealed factor structures and the Cronbach alpha values of the subscales were consistent with original versions.

\section{Conclusions}

The Sri Lankan version of BIS/BAS, SPSRQ and SSS scales demonstrated adequate psychometric properties to be used in future studies.

SL J Psychiatry 2011; 2 (1):13-17

\section{Introduction}

People react differently to similar situations; this is the fundamental difference between people which we refer to as "personality". Personality may influence one's vulnerability to disorders, health-seeking behaviours and treatment compliance. A growing body of research provides evidence for the role of personality in certain mental health disorders including bipolar disorders (1), schizophrenia, substance abuse disorders $(2,3)$ and eating disorders (4) as well as non-psychiatric diseases such as coronary artery disease (5), inflammatory bowel disease (6) peptic ulcer disease (7) and sleep disorders (8).

Gray's theory of personality has been one of the most researched and reviewed (9). This incorporates three motivational systems. The Behavioural Inhibition System (BIS), which is neurobiologically related to the septohippocampal region and its neural connections to the forebrain, controls aversive behaviour in response to punishment and non-reward. Increased BIS sensitivity leads to anxiety related traits. The Behavioural Activation System (BAS), which is linked to the mesolimbic reward system, on the other hand controls approach behavior in response to rewarding stimuli. Heightened sensitivity to BAS is associated with impulsive behaviour. The third system, the Fight and Flight system (FFS), according to Gray's original theory, controls behavior in response to unconditioned aversive stimuli.

Over the years, a number of self-report measures have been developed to assess Gray's theory. However these measures have only considered BIS and BAS as the two main systems controlling behaviour. Carver and White's BIS/BAS scales are the most frequently used and researched (10). The BAS has three subscales including BAS drive (BAS-Drv) (persistence in obtaining desired goals), BAS fun-seeking (BAS-FS) (willingness to seek out and spontaneously approach potentially rewarding experiences) and BAS reward responsiveness (BAS-RR) (anticipation and positive response towards reward) components.

The BIS scale elicits respondents' reactions to cues of impending punishment. The BIS/BAS questionnaire comprises of 20 scored items and four filler items. All these areas are scored using four-point Likert-type scales that derive a global BIS score and three BAS related subscale scores. Therefore, broadly the BAS score can be considered as a measure of impulsivity while the BIS score is a measure of anxiety-related traits.

Another measure of personality, based on Gray's theory, is the Sensitivity to Punishment and Sensitivity to Reward Questionnaire (SPSRQ)(11). This questionnaire consists of two separate scales with Sensitivity to Punishment (SPSRQ-SP) measuring the BIS and Sensitivity to Reward (SPSRQ-SR) measuring the BAS. The original questionnaire consisted of 48 items. However, following subsequent psychometric studies, the item number was trimmed down to 35 with 17 items measuring SP and 18 items measuring SR.

Another prominent theory of personality is the sensation-seeking theory $(12,13)$. In essence this 
proposes that an individual needs a distinctively determined, homeostatic degree of stimulation with which one feels comfortable. If the environment is unable to provide this optimal level of stimulation, the individual will tend to seek complexity or novelty, to the extent of compensating for the sub-optimal level of stimution which may vary among individuals. In an attempt to get optimal arousal, the individual could engage in either risky or non-risky behaviours. Sensation-seeking behaviour generally peaks during late adolescence and gradually diminishes with age. However, low and high sensation-seekers remained distinct and stable in a cohort, irrespective of the age (13, 14). Sensation-seeking is measured by the Zuckerman's Sensation Seeking Scale (SSS), which includes four subscales; Thrill and Adventure Seeking (TAS), Experience Seeking (ES), Disinhibition (DIS) and Boredom Susceptibility (BS). TAS assesses the desire for and physical engagement in speedy and dangerous events. ES reflects the need for novel experiences such as exploring new destinations and interactions with different cultures. DIS assesses the lack of social restraint and the tendency to seek hedonistic social orientation such as sexual promiscuity. BS reflects the propensity to have an aversion to routine events and impatience due to lack of stimulus diversity. Initially, there were 113 items in the SSS but the latest version (Form V) consists only of 40 items with 10 items for each subscale. The reliability and the construct validity of this tool have been well established in both clinical and non-clinical samples $(13,15,16)$. Many different versions of the SSS have been since developed including a shorter version, a sexual sensation-seeking scale and an impulsive sensation-seeking scale which is linked to monoaminergic transmission (17-19). Recently sensation-seeking has been genetically linked to a number of dopaminergic gene polymorphisms (20). Both Gray's Personality theory and Zuckerman's Sensation-seeking theory have been used to explain normal personality as well as psychopathology. Bipolar disorders have been shown to be associated with both elevated BIS and BAS; in particular, high BAS scores have been associated with more manic symptoms. High BIS sensitivity was linked to anxiety disorders (21) and internalising behaviours (22). Furthermore, the use of behavioural activation interventions during cognitive behaviour therapy has been shown to be successful in improving depression (23). In addition, impulsivity has been identified as a major factor associated with initiation and progression of substance abuse and addiction and may account for $30-60 \%$ of overall risk for developing addictive disorders. BAS-Drv and BASFS are positively correlated with alcohol use and abuse (24). Further, a community-based study showed that high scores in the BAS-RR and BAS-FS components are associated with the presence of a lifetime alcohol abuse disorder (25). Similarly sensation-seeking has been shown to be associated with psychopathy (26), substance abuse and dependence $(27,28)$, alcohol abuse and dependence (29), substance dependence associated with major psychiatric disorders (30), pathological gambling (31) and motor vehicle accidents $(31,32)$.

The value of personality inventories in both research and clinical practice has been widely accepted. There is more than sufficient evidence to suggest how personality as measured by BIS/BAS or SSS can influence the development, progression, clinical evaluation and management of psychopathological conditions. At present research and clinical utilisation of personality measures in Sri Lanka has been minimal largely due to lack of translated and validated assessment tools. The aim of the present study is to translate and assess the psychometric properties of a Sinhalese adaptation of the BIS/BAS, SPSRQ and SSS scales in a Sri Lankan population.

\section{Methods}

Ethical clearance for the study was obtained from the committee of research and ethical review, Faculty of Medicine, University of Peradeniya, Sri Lanka. Two hundred and thirty three Sri Lankan university students ( $72 \%$ female) were recruited for the study. The mean age of the participants was $22.8 \pm 2.6$ years.

\section{Measures}

The BIS/BAS, SPSRQ and SSS scales were translated into Sinhalese by experts in Psychiatry, Sociology and English. The translated Sinhalese versions were then checked for wording and content and the draft version of the Sinhalese BIS/BAS, SPSRQ and SSS were prepared. The drafts were then back-translated into English by an expert translator. The original English versions and the back-translated English versions were then evaluated for convergence, following which the final versions of the Sinhalese BIS/BAS, SPSRQ and SSS were prepared. The final version of the Sinhalese version of BIS/BAS consisted of the original 20 scale items and four 'filler' items. Only the 20 scale items were used in the following analysis. As in the original, each item was scored using a four-point Likert scale, ranging from 1 (strongly disagree) to 4 (strongly agree). The Sri Lankan versions of SPSRQ and SSS comprised 35 and 40 items with binary (Yes/No) type responses respectively.

\section{Procedure}

The BIS/BAS, SSS and SPSRQ questionnaires were given to the participants to complete in large groups under the direct supervision of a team of research assistants.

\section{Statistical Analyses}

The model fits of BIS/BAS, SPSRQ and SSS were assessed using confirmatory factor analysis (CFA) performed using AMOS 18 statistical software (37). The basic four factor model of the BIS/BAS and the two factor models of SPSRQ and SSS were evaluated. Since SPSRQ and SSS comprise of binary response items, item parceling was done before the CFA. According to Nasser and Takahashi (36), solutions from parceled data with more items per parcel result in more normality, validity, continuity, and reliability than from parceled data with fewer items per parcel. Therefore we included three parcels comprising 6, 6 and 5 items for the SP subscale of the SPSRQ and 6, 5 and 5 items for the SR subscale of the SPSRQ. Each subscale of SSS was assigned to two parcels each comprising 5 items. During the CFA all factors were allowed to co-vary. Covariance matrices were analysed using maximum 
likelihood estimation. The chi-square $(\chi 2)$ goodnessof-fit index statistic, root mean squared error of approximation (RMSEA), comparative fit index (CFI) and standardized root mean residual (SRMR) were used to evaluate model fit. For a model to be considered to have an acceptable fit, RMSEA and SRMR should be $<0.06$ and $<0.08$ respectively, with a CFI $>0.90$ (38).

\section{Results}

\section{Data screening}

There was no missing data in this sample. Calculation of the Mahalanobis distance revealed no multivariate outliers.

\section{Descriptive Statistics}

Significant gender differences were observed for subscale scores SPSRQ-SP, BAS -RR, SSS-TAS, SSSDIS and SSS-ES with females recording higher BIS scores and males recording higher sensation-seeking and reward-sensitivity scores (Table 1).

\section{CFA Models}

The results of the CFA are shown in Table 2. The tested models demonstrated acceptable fit for the data according to multiple fit indices. The chi-square value for BIS/BAS was statistically significant but not for SPSRQ and SSS. The unstandardized regression weight of the BIS item "I have very few fears compared to my friends" was not statistically significant and the standardized regression weight was very low. All the standardized regression weights of item parcels of subscales of both SPSRQ and SSS were acceptable. Internal Consistency

As a measure of reliability, Cronbach's alphas for the BAS-drive, fun-seeking, reward-sensitivity subscales and the BIS subscales were $0.73,0.82,0.80$ and 0.65 respectively. Deletion of the BIS item "I have very few fears compared to my friends" improved the alpha value of the BIS scale to 0.70 . For the SPSRQ, an alpha

\begin{tabular}{|c|c|c|c|}
\hline Subscale & Gender & Number & Mean $\pm S D$ \\
\hline \multirow[t]{2}{*}{ BAS-DR } & female & 168 & $10.6 \pm 2.1$ \\
\hline & male & 65 & $11.1 \pm 2.2$ \\
\hline \multirow[t]{2}{*}{ BAS-FS } & female & 168 & $9.9 \pm 2.5$ \\
\hline & male & 65 & $10.1 \pm 2.4$ \\
\hline \multirow[t]{2}{*}{ BAS-RR } & female & 168 & $15.1 \pm 2.4$ \\
\hline & male & 65 & $16.0 \pm 2.5^{*}$ \\
\hline \multirow[t]{2}{*}{ BIS } & female & 168 & $20.6 \pm 2.8$ \\
\hline & male & 65 & $20.2 \pm 2.9$ \\
\hline \multirow[t]{2}{*}{ SSS-TAS } & female & 168 & $3.0 \pm 2.3$ \\
\hline & male & 65 & $5.5 \pm 3 * * *$ \\
\hline \multirow[t]{2}{*}{ SSS-BS } & female & 168 & $2.1 \pm 1.9$ \\
\hline & male & 65 & $1.9 \pm 1.4$ \\
\hline \multirow[t]{2}{*}{ SSS-ES } & female & 168 & $1.7 \pm 1.6$ \\
\hline & male & 65 & $2.8 \pm 1.6 * * *$ \\
\hline \multirow[t]{2}{*}{ SSS-DIS } & female & 168 & $2.0 \pm 1.8$ \\
\hline & male & 65 & $3.2 \pm 2.1 * * *$ \\
\hline \multirow[t]{2}{*}{ SPSRQ SR } & female & 168 & $8.0 \pm 3.7$ \\
\hline & male & 65 & $8.4 \pm 2.8$ \\
\hline \multirow[t]{2}{*}{ SPSRQ SP } & female & 168 & $10.5 \pm 3.5^{* * *}$ \\
\hline & male & 65 & $8.5 \pm 3.7$ \\
\hline
\end{tabular}

\begin{tabular}{|c|c|c|c|c|c|}
\hline Score & $x^{2}$ & df & SRMR & $\mathrm{CFI}$ & RMSEA \\
\hline BIS/BAS 4 Factor & $277.4^{*}$ & 164 & 0.07 & 0.91 & 0.05 \\
\hline SPSRQ 2 Factor & 10.05 & 8 & 0.03 & 0.99 & 0.03 \\
\hline SSS 4 Factor & 28.4 & 14 & 0.03 & 0.98 & 0.06 \\
\hline
\end{tabular}

$* p>0.05$

value of 0.73 was found for both SP and SR subscales. The alpha values of SSS subscales TAS, ES, DIS and BS were $0.80,0.62,0.80$ and 0.62 respectively. The alpha value for the SSS was 0.88 .

\section{Concurrent Validity}

The inter-correlations among the subscales are shown in Table 3. As expected BIS and SPSRQ-SP subscales are positively correlated. Both BIS and SPSRQ-SP are negatively correlated with all BAS scales, SPSRQSR and SSS subscales except SSS-BS. The BAS subscales of BIS/BAS, SR subscale of SPSRQ and the subscales of SSS are positively correlated except for the significantly negative correlation between BIS-RS and SSS-BS.

\section{Discussion}

In this study we translated and assessed the psychometric properties of a Sinhalese adaptation of the BIS/BAS, SPSRQ and SSS scales in a Sri Lankan population. Results showed that the psychometric properties of the Sinhalese versions of BIS/BAS, SPSRQ and SSS scales were sound and in line with the original English versions and other translated versions.

According to the CFA results, the four factor model of the BIS/BAS scale demonstrated an adequate fit for the data as indicated by multiple fit indices despite the chi-square test being significant. It is well known that the chi-square statistic is very sensitive with large sample sizes. For this reason, a number of fit indices were used to assess the overall model fit (33). Previous research also provides support for a four factor model of BIS/BAS $(34,35)$. The reversed-scored BIS item, "I have very few fears compared to my friends" was not significant and had poor factor loading. Similar findings for this item have been reported in other studies $(36,37,38,39)$ which found this item (and the other reverse-scored BIS items) problematic in their studies involving adolescent, clinical and offender samples. Poythress and colleagues (39) suggested this item and the other reverse-scored BIS item "Even if something bad is about to happen to me, I rarely experience fear or nervousness" may explore a different aspect of behavioural inhibition. Another reason may be that this particular item may not be relevant to the Sri Lankan culture.

While previous studies have reported the BAS-RR $(24,35)$ or BAS-FS scales $(40)$ as the least internally consistent of the BIS/BAS scales, in the Sri Lankan version, the BIS scale had the lowest alpha value. This may be partly due to the inclusion of the BIS item "Even if something bad is about to happen to me, I rarely experience fear or nervousness", as removal of this item improved the alpha values. 


\begin{tabular}{|c|c|c|c|c|c|c|c|c|c|}
\hline & BAS-drv & BAS-FS & BAS-RS & BIS & SSS-TAS & SSS-BS & SSS-ES & SSS-DIS & $\begin{array}{l}\text { SPSRQ } \\
\text { SR }\end{array}$ \\
\hline \multicolumn{10}{|l|}{ BAS-drv } \\
\hline BAS-FS & $.269 * *$ & & & & & & & & \\
\hline BAS-RS & $-.339 * *$ & .095 & & & & & & & \\
\hline BIS & -.046 & .017 & $.235^{* *}$ & & & & & & \\
\hline SSS-TAS & $.225^{* *}$ & $.261^{* *}$ & .052 & $-.206^{* *}$ & & & & & \\
\hline SSS-BS & .061 & $.389 * *$ & $-.175^{* *}$ & $-.135^{*}$ & $.440 * *$ & & & & \\
\hline SSS-ES & .123 & $.330 * *$ & -.055 & $-.138 *$ & $.547^{* *}$ & $.542^{* *}$ & & & \\
\hline SSS-DIS & .100 & $.204^{* *}$ & .064 & $-.149 *$ & $.469 * *$ & $.323 * *$ & $.428 * *$ & & \\
\hline SPSRQ-SR & $.156^{*}$ & $.187^{* *}$ & .010 & $-.134 *$ & $.200 * *$ & $.447^{* *}$ & $.274^{* *}$ & $.271^{* *}$ & \\
\hline SPSRQ-SP & -.123 & .075 & -.078 & $.264 * *$ & $-.204 * *$ & $.162^{*}$ & -.073 & -.077 & $.194 * *$ \\
\hline
\end{tabular}

Similar to the BIS/BAS scale, the SPSRQ, which is the other tool based on Gray's theory of personality, recorded acceptable multiple fit indices suggesting that the test model of SPSRQ fits our data. In addition, the chi-square test was also non-significant, further strengthening the model fit. All the item parcels created for the subscales of both SPSRQ and SSS had acceptable factor loadings. The Cronbach alpha values of SPSRQ-SP and SPSRQ-SR were in agreement with previous studies indicating satisfactory reliability and internal consistency among the items of the two scales $(11,41)$. The positive correlations between BIS and SPSRQ-SP (that are measures of behavioural inhibition) and between the BAS subscales and SR (that are measures of approach behaviour), indicate the concurrent validity of the BIS/BAS and SPSRQ scales.

The Sinhalese version of the SSS also demonstrated satisfactory psychometric properties as evidenced by the results of the CFA supporting a four factor model consistent with previous studies $(12,42)$. Although the TAS and BS scales recorded ordinary alpha values, they were comparable to the original English version of SSS (12) and better than some of the translated versions (43). Conceptually, sensation-seeking relates to BAS (14). Accordingly SSS subscales were positively correlated with both BAS subscales and the SR component of the SPSRQ scale except for the negative correlation of SSS-BS with BAS-RS. A reason for this may be the positive relationship between BAS-RS and BIS, a finding frequently reported and replicated $(10,36)$.

Overall, the Sri Lankan version of the BIS/BAS, SPSRQ and SSS scales demonstrated adequate psychometric properties to be used in future studies. It would however be necessary to use a larger sample representing different sections of the community to further validate and refine these tools to elucidate any ethnic variation if present.

\section{Acknowledgment}

We are grateful to all participants who voluntarily engaged in this survey and those who helped in the translation process of BIS/BAS, SSS and SPSRQ questionnaires.

Note: The Sinhalese versions of the questionnaires are available from the authors on request.

\section{Declaration of interest}

None

Lakal Dissabandara, Lecturer, School of Medicine, University of Queensland, Australia and Faculty of Medicine, University of Peradeniya, Sri Lanka

Natalie Loxton, Lecturer, School of Psychology,

The University of Queensland, Australia

Shavindra Dias, Lecturer, Faculty of Medicine, University of Peradeniya, Sri Lanka

Mark Daglish, Senior Lecturer, School of Medicine,

University of Queensland, Australia

Alfreda Stadlin, Professor, Chungbuk National University, South Korea

Corresponding author

Shavindra Dias, Faculty of Medicine, University of

Peradeniya, Sri Lanka

E mail: shavindra17@yahoo.co.uk

\section{References}

1. Swann AC, Pazzaglia, P., Nichols, A., Dougherty, D.M., \& Moeller, F.G. . Impulsivity and phase of illness in bipolar disorders. Journal of Affective Disorders. 2003;77:105-11.

2. Hussong AM, \& Chassin, L.The stress-negative affect model of adolescent alcohol use:disaggregating negative affect. Journal of studies on alcohol. 1994;55:707-18.

3. Baker TB, Brandon, T.H., \& Chassin, L. . Motivational influences on cigarette smoking. Annual Review of Psychology. 2004;55:463-91.

4. Loxton NJ, Sharon D. Alcohol abuse and dysfunctional eating in adolescent girls: The influence of individual differences in sensitivity to reward and punishment. International Journal of Eating Disorders. 2001;29:455-62.

5. Miller TQ, Smith, T. W., Turner, C. W., Guijarro, M.L., \& Hallett, A. J. . A meta-analytic review of research on hostility and physical health. Psychological Bulletin. 1996;119:32248.

6. Robertson DAF, Ray, J., Diamond, I., \& Edward,J. G.. Personality profile and affective state of patients with inflammatory bowel disease. Gut. 1989;30:623-6.

7. Feldman M, Walker, P., Goldschmiedt, M., \& Cannon, D. Role of affect and personality in gastric acid secretion and serum gastrin concentration: Comparative studies in normal men and in male duodenal ulcer patients. Gastroenterology. 1992;102:175-80. 
8. Ireland J, \& Culpin, V. . The relationship between sleeping problems and aggression, anger, and impulsivity in a population of juvenile and young offenders. Journal of Adolescent Health. 2006;38:649-55.

9. Gray JA. The psychophysiological basis of introversionextraversion. Behav Res Ther. 1970 Aug;8:249-66.

10. Carver CS, White TL. Behavioral inhibition, behavioral activation, and affective responses to impending reward and punishment:The BIS/BAS Scales. Journal of personality and social psychology. 1994;67: 319-33.

11. Torrubia R, Ávila C, Moltó J, Caseras X. The Sensitivity to Punishment and Sensitivity to Reward Questionnaire (SPSRQ) as a measure of Gray's anxiety and impulsivity dimensions. Personality and Individual Differences. 2001;3:837-62.

12. Zuckerman M, Eysenck, S., Eysenck, H. J. . Sensation seeking in England and America: cross-cultural, age, and sex comparisons. J Consult Clin Psychol. 1978;46:139-49.

13. Zuckerman M. Behavioral expression and biosocial bases of sensation seeking. New York Cambridge University Press; 1994.

14. Zuckerman M, editor. sensation seeking: beyond the optimal level of arousal. Hillsdale, NJ: Lawrence Erlbaum Associates Inc; 1979.

15. Loas G, Verrier A, Flament MF, Perez-Diaz F, Corcos M, Halfon $\mathrm{O}$, et al. Factorial structure of the Sensation-Seeking Scale-Form V: Confirmatory factorial analyses in nonclinical and clinical samples. Canadian Journal of Psychiatry Clin Neurosci. 2001;46:850-5.

16. Roberti JW, Storch EA, Bravata E. Further psychometric support for the Sensation Seeking Scale - Form V. Journal of Personality Assessment. 2003;81:291-2.

17. Netter P, Hennig J, Roed IS. Serotonin and dopamine as mediators of sensation seeking behavior Neuropsychobiology. 1996;34:155-65.

18. Gerra G, Avanzini P, Zaimovic A, Sartori R, Bocchi C, Timpano $\mathrm{M}$, et al. Neurotransmitters, neuroendocrine correlates of sensation-seeking temperament in normal humans. Neuropsychobiology. 1999;39:207-13.

19. Zuckerman M. The Neurobiology of Impulsive Sensation Seeking: Genetics, Brain Physiology, Biochemistry, and Neurology. 2005. p. 31-52.

20. Derringer J, Krueger RF, Dick DM, Saccone S, Grucza RA, Agrawal A, et al. Predicting Sensation Seeking From Dopamine Genes. Psychological Science. 2010 September 1, 2010;21:1282-90.

21. Carver CS. Negative Affects Deriving From the Behavioral Approach System. Emotion. 2004;4:3-22.

22. Colder C, O'Connor R. Gray's Reinforcement Sensitivity Model and Child Psychopathology: Laboratory and Questionnaire Assessment of the BAS and BIS. Journal of Abnormal Child Psychology. 2004;32:435-51.

23. Jacobson NS, Dobson, K S., Truax, P. A., Addis, M. E., Koerner, K.,Gollan, J. K. A component analysis of cognitivebehavioral treatment for depression. Journal of Consulting and Clinical Psychology. 1996;64:295-304.

24. Jorm AF, Christensen H, Henderson AS, Jacomb PA, Korten AE, Rodgers B. Using the BIS/BAS Scales to measure behavioural inhibition and behavioural activation: Factor structure, validity and norms in a large community sample. Personality and Individual Differences. 1999;26:49-58.

25. Johnson SL, Turner RJ, Iwata N. BIS/BAS Levels and Psychiatric Disorder: An Epidemiological Study. Journal of Psychopathology and Behavioral Assessment. 2003;25:2536.
26. Blackburn R. Sensation seeking, impulsivity, and psychopathic personality. Journal of Consulting and Clinical Psychology. 1969;33:571-4.

27. Franques P, Auriacombe, M., Piquemal, E.; Verger, M., Brisseau-Gimenez, S.,Grabot, D. \& Tignol, J. Sensation seeking as a common factor in opioid dependent subjects and high risk sport practicing subjects. A cross sectional study. Drug Alcohol Depend. 2003; 69:121-6.

28. Gonzalez R, Vassileva J, Bechara A, Grbesic S, Sworowski L, Novak RM, et al. The influence of executive functions, sensation seeking, and HIV serostatus on the risky sexual practices of substance-dependent individuals. J Int Neuropsychol Soc. 2005 Mar;11:121-31.

29. Hittner JB, Swickert R. Sensation seeking and alcohol use: A meta-analytic review. Addictive Behaviors. 2006;31:1383401.

30. Dervaux A, Laqueille X, Bourdel M-C, Oli? J-P, Krebs M-O. Impulsivity and sensation seeking in alcohol abusing patients with schizophrenia. Frontiers in Psychiatry. [Original Research]. 2010 2010-October-19;1:12.

31. Coventry KR, Brown RIF. Sensation seeking, gambling and gambling addictions. Addiction. 1993;88(4):541-54.

32. Dunlop SM, Romer D. Adolescent and Young Adult Crash Risk: Sensation Seeking, Substance Use Propensity and Substance Use Behaviors. Journal of Adolescent Health. 2010;46:90-2.

33. Stevens J, editor. Applied multivariate statistics for the social sciences. 3 ed. Mahwah, NJ: Lawrence Erlbaum Associates; 1996.

34. Heubeck BG, Wilkinson RB, Cologon J. A second look at Carver and White's BIS/BAS Scales (1994). Personality and Individual Differences. 1998;25:785-800.

35. Müller JM, Wytykowska AM. Psychometric properties and validation of a Polish adaptation of Carver and White's BIS/BAS scales. Personality and Individual Differences. 2005;39:795-805.

36. Campbell-Sills L, Liverant GI, Brown TA. Psychometric Evaluation of the Behavioral Inhibition/Behavioral Activation Scales in a Large Sample of Outpatients With Anxiety and Mood Disorders. Psychological Assessment. 2004; 16:244-54.

37. Cogswell A, Alloy LB, Van Dulmen MHM, Fresco DM. A psychometric evaluation of behavioral inhibition and approach self-report measures, Personality and Individual Differences. 2006;40:1649-58.

38. Cooper A, Gomez R, Aucote H. The Behavioural Inhibition System and Behavioural Approach System (BIS/BAS) Scales: Measurement and structural invariance across adults and adolescents. Personality and Individual Differences. 2007;43:295-305.

39. Poythress NG, Skeem JL, Weir J, Lilienfeld SO, Douglas KS, Edens JF, et al. Psychometric Properties of Carver and White's (1994) BIS/BAS Scales in a Large Sample of Offenders. Pers Individ Dif. 2008 Dec;45:732-7.

40. Franken IHA, Muris P, Rassin E. Psychometric Properties of the Dutch BIS/BAS Scales. Journal of Psychopathology and Behavioral Assessment. 2005;27.

41. Brunelle C, Douglas RL, Pihl RO, Stewart SH. Personality and substance use disorders in female offenders: A matched controlled study. Personality and Individual Differences. 2009;46:472-6.

42. Aluja A, Garcia O, GarcíA LF. Exploring the structure of Zuckerman's sensation seeking scale, form $\mathrm{V}$ in a spanish sample. Psychological Reports. 2004 2004/08/01;95:338-44.

43. Loxton NJ, Wan VL, Ho AM, Cheung BK, Tam N, Leung FY, et al. Impulsivity in Hong Kong-Chinese club-drug users. Drug and Alcohol Dependence. 2008;95:81-9. 